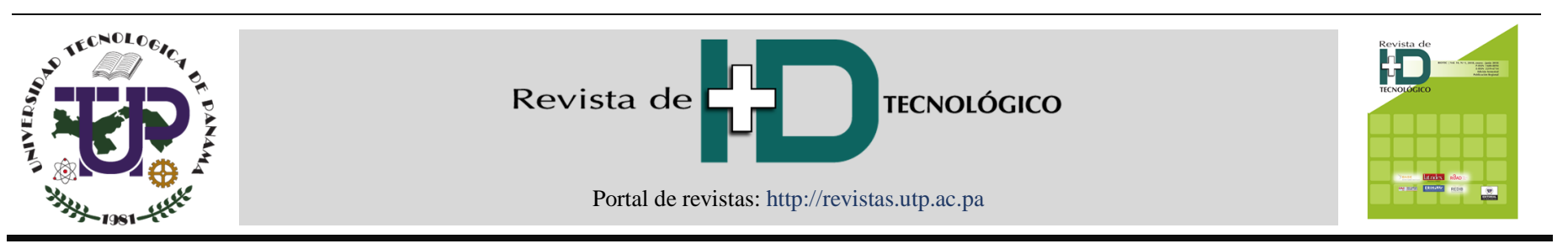

\title{
Modelado de la isoterma de adsorción de zanahorias deshidratadas
}

\section{Modelling of moisture sorption isotherms of dehydrated carrot}

\author{
Marialina Anria $^{1 *}$, Astrid González', Rosa I. Quintero ${ }^{2}$ \\ ${ }^{1}$ Facultad de Ciencias y Tecnología, Licenciatura de Ingeniería en Alimentos, Universidad Tecnológica de Panamá, Panamá \\ ${ }^{2}$ Ciencia y Tecnología Innovadora en Alimento (CYTIA), Facultad de Ciencias y Tecnología, Universidad Tecnológica de Panamá, Panamá \\ *Autor de correspondencia: maria-lina-23@hotmail.com
}

\begin{abstract}
RESUMEN-El comportamiento de las isotermas de adsorción es importante ya que estos garantizan la estabilidad de los productos alimenticios durante los procesos de secado, envasado y almacenamiento. En este estudio se determinó a una temperatura de $22^{\circ} \mathrm{C} \pm 1{ }^{\circ} \mathrm{C}$ la isoterma de sorción de zanahoria aplicando el método de gravimetría estática en un rango de actividad de agua (Aw) entre 0.113 y 0.883 . Los datos se ajustaron utilizando los modelos matemáticos GAB, BET y Henderson y la calidad del ajuste se evaluó con el coeficiente de regresión ( $\mathrm{R}^{2}$ ). Se obtuvo una isoterma tipo II, la cual es típica para frutas y vegetales. La humedad de la monocapa (Wo) fue ligeramente menor en el modelo de BET que en el de GAB, con valores de 0.102 y $0.125 \mathrm{~g}$ agua/g de m.s., respectivamente, lo cual concuerda con la literatura consultada. Todos los modelos presentaron un ajuste superior a 0.92. Sin embargo, los que mejor se ajustaron al comportamiento de los datos experimentales fue de BET y GAB, los cuales presentaron un $\mathrm{R}^{2}$ de 0.975 y 0.994 , respectivamente.
\end{abstract}

Palabras clave-Actividad de agua, deshidratado, humedad relativa, isoterma, modelado matemático, sorción, zanahoria.

ABSTRACT-The behavior of the adsorption isotherms is important since these guarantee the stability of the food products during the drying, packaging and storage processes. In this study, the carrot sorption isotherm was determined at a temperature of $22^{\circ} \mathrm{C} \pm 1{ }^{\circ} \mathrm{C}$ applying the method of static gravimetry method in a range of water activity (Aw) between 0.113 and 0.883 . The data were adjusted using the GAB, BET and Henderson mathematical models and the quality of the adjustment was evaluated with the regression coefficient $\left(\mathrm{R}^{2}\right)$. An isotherm type II was obtained, which is typical for fruits and vegetables. The humidity of the monolayer (Wo) was slightly lower in the BET model than in that of GAB, with values of 0.102 and $0.125 \mathrm{~g}$ water / $\mathrm{g}$ of m.s, respectively, which agrees with the literature consulted. All models presented an adjustment higher than 0.92 . However, those that best adjusted to the behavior of the experimental data were BET and GAB, which presented an $\mathrm{R}^{2}$ of 0.975 and 0.994 , respectively.

Keywords-Water activity, dehydrated, relative humidity, isotherm, math model, sorption, carrot.

\section{Introducción}

La zanahoria (Daucus carota L.) es un producto que se produce a lo largo de todo el año en las tierras altas de Panamá [1] que se consume en forma fresca, procesada y deshidratadas en sopas o encurtidos [2] .

Se encuentra dentro de las hortalizas de raíz más populares al ser una de las fuentes más rica de carotenoides $(11,263 \mathrm{ug} / 100 \mathrm{~g})$ y fibra $(2.8 \mathrm{~g} / 100 \mathrm{~g})$ [3] [4].

Los carotenoides son metabolitos secundarios obtenidos de las plantas, a los cuales se les atribuye actividades antioxidantes y pueden controlar o prevenir enfermedades inducidas por radicales libres [5].

Las zanahorias crudas sufren deterioro microbiológico y bioquímico debido a ser un alimento perecedero con alto contenido de agua. Cuando son procesadas térmicas mediante cocción, esterilización y deshidratado se puede afectar el contenido de carotenoide, el color y textura debido al incremento de procesos de oxidación [6].

Relacionar el contenido de humedad y la correspondiente actividad de agua a una temperatura determinada, se obtienen las isotermas de sorción de un producto; estas, reflejan un equilibrio entre el producto y su atmósfera. Proveen información acerca de la distribución y las formas de agua que están presentes en el producto, la intensidad de las uniones y disponibilidad de la misma [7].

En base al modo de obtención, se clasifican en isotermas de adsorción y desorción. En las primeras, se coloca la muestra seca en atmósferas de humedad relativa superior a su actividad de agua para que haya una

Citación: M. Anria, A. González y R. Quintero, “Modelado de la isoterma de adsorción de zanahorias deshidratadas”, Revista de I+D Tecnológico, vol. 15, no. 1, pp. (17-23), 2019.

Tipo de artículo: Original. Recibido: 22 de julio de 2018. Recibido con correcciones: 20 de agosto de 2018. Aceptado: 19 de noviembre de 2018 .

DOI https://doi.org/10.33412/idt.v15.1.2093

Copyright: 2019 M. Anria, A. González y R. Quintero. This is an open access article under the CC BY-NC-SA 4.0 license (https://creativecommons.org/licenses/bync-sa/4.0/). 
adsorción de agua y en las segundas, se coloca la muestra húmeda en atmósferas de humedad relativa inferior a su actividad de agua de modo que pierdan agua. En ambas, el equilibrio se alcanza cuando el peso de la muestra se mantiene constante. Sin embargo, las isotermas de adsorción y desorción para un mismo producto no suelen coincidir. La diferencia entre las curvas se observa en la figura 1, donde la isoterma de adsorción se sitúa por debajo de la desorción. La región que se encuentra entre ambas se define como histéresis.

Las isotermas se dividen en tres regiones; la región A representa la zona de baja Aw donde se encuentra el agua ligada, que incluye el agua estructural y de la monocapa. Esta agua no es congelable y no está disponible para reacciones químicas. En la región B el agua está unida más débilmente que en la región anterior y usualmente se presenta en pequeños capilares; a esta, se le conoce como zona de Aw intermedia. El agua de la región $\mathrm{C}$, conocida como zona de Aw alta, es el agua libre que está encerrada en tejidos y membranas o contenida en macrocapilares [8].

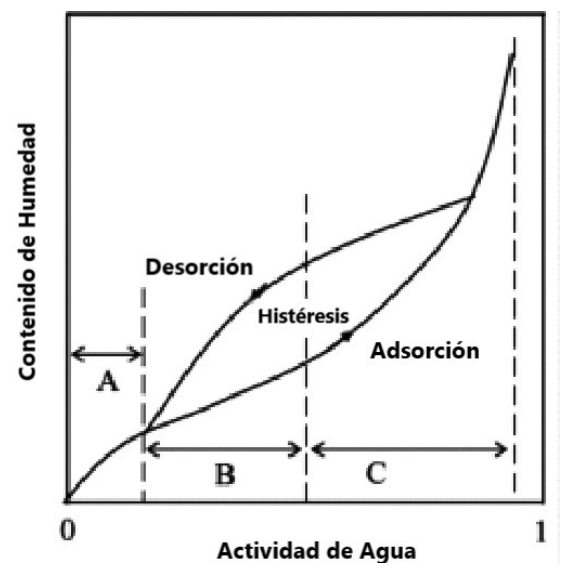

Figura 1. Isoterma de sorción típica de un producto [8].

Las isotermas de adsorción se utilizan en cuatro áreas del procesamiento de alimentos: secado, mezclado, envasado y almacenamiento [9].

La modelización de las isotermas de sorción es posterior al proceso de secado. Debido a la complejidad de los alimentos, no hay ningún modelo que se ajuste en todos los casos. Por lo tanto, cuando se aborda la modelización de un producto no estudiado previamente se debe evaluar la capacidad de ajuste de diferentes modelos con el objetivo de disponer para la optimización de distintas ecuaciones con diferente grado de aplicabilidad [10].

El objetivo de este estudio fue obtener la isoterma de adsorción de humedad a una temperatura de $22^{\circ} \mathrm{C}$ de la zanahoria (Daucus carota L.) deshidratada y posteriormente ajustada mediante los modelos matemáticos de BET, GAB y Henderson.

\section{Materiales y Métodos}

\subsection{Obtención de la materia prima}

Las zanahorias fueron adquiridas en un supermercado de la localidad. Para la elección se tuvo en consideración tamaño, forma y uniformidad.

\subsection{Preparación de la muestra y deshidratado}

Las muestras fueron lavadas, peladas, pesadas $\mathrm{y}$ cortadas en rodajas de $3-5 \mathrm{~mm}$ de espesor. Posteriormente, colocadas en bandejas en el deshidratador de aire de temperatura controlada marca Presto, a una temperatura de $46^{\circ} \mathrm{C}$ por 20 horas. Al finalizar se pesaron y se colocaron en bolsas de polietileno selladas para evitar la absorción de humedad del ambiente.

\subsection{Determinación de la isoterma}

La determinación de la humedad de equilibrio se realizó utilizando el método estático de gravimetría a temperatura de $22^{\circ} \mathrm{C} \pm 1^{\circ} \mathrm{C}$.

El diseño experimental consistió en colocar una masa conocida de muestra (por triplicado) en recipientes cerrados herméticamente (desecadores), los cuales contenían soluciones de sales saturadas que proporcionaban diferentes humedades relativas. En el equilibrio, la actividad de agua (aw) es igual a la humedad relativa del aire que rodea al producto a una temperatura determinada. Generalmente se trabaja con valores de aw conocida $(0-1)$ de acuerdo a las sales utilizadas. El valor de las actividades de agua utilizada en este estudio se muestra en la tabla 1. A cada desecador se le agregaron $150 \mathrm{~mL}$ de solución saturada en el fondo y la muestra se colocó en la bandeja superior en cápsulas de porcelana. En la figura 2 se muestra el sistema utilizado. 
Tabla 2. Ecuaciones para el modelado de las isotermas de sorción

\begin{tabular}{|l|c|l|}
\hline Modelo & \multicolumn{1}{|c|}{ Ecuación } & \multicolumn{1}{c|}{ Variables } \\
\hline BET & $W e=\frac{W_{0} \cdot C \cdot A w}{(1-A w) \cdot(1(C-1) \cdot A w)}$ & $\begin{array}{l}W_{o}: \text { humedad del producto correspondiente } \\
\text { a una capa monomolecular de agua } \\
\text { adsorbida. } \\
C: \text { constante característica del material } \\
\text { relacionada con el calor desprendido en el } \\
\text { proceso de sorción. }\end{array}$ \\
\hline GAB & $W e=\frac{W_{o} \text { humedad del producto correspondiente }}{(1-K \cdot A w) \cdot(1+(C-1) \cdot K \cdot A w)}$ & $\begin{array}{l}W_{0} \cdot C \cdot K \cdot A w \\
\text { la situación en que los puntos de adsorción } \\
\text { primarios están saturados por moléculas de } \\
\text { agua. } \\
C: \text { constante de Guggenheim, característica } \\
\text { del producto y relacionada con el calor de } \\
\text { adsorción de la monocapa. } \\
\text { K: factor de corrección relacionado con el } \\
\text { calor de sorción de la multicapa. }\end{array}$ \\
\hline Henderson & $W e=0.01\left(\frac{-\log (1-A w)}{10^{f}}\right)^{1 / n}$ & $\begin{array}{l}n \text { y } f \text { son parámetros característicos del } \\
\text { producto. }\end{array}$ \\
\hline Smith & $W e=B+A \cdot \ln (1-A w)$ & $\begin{array}{l}A \text { y } B: \text { constantes del modelo y } \\
\text { características para cada alimento. }\end{array}$ \\
\hline Oswin & $W e=C\left(\frac{A w}{1-A w}\right)^{n}$ & $\begin{array}{l}\text { C y } n: \text { constantes del modelo y } \\
\text { características para cada alimento. }\end{array}$ \\
\hline
\end{tabular}

Fuente: Navarrete [11] y Zapata [12]

Tabla 1. Soluciones de sales saturadas, humedad relativa (\%HR) y su respectiva actividad de agua (Aw)

\begin{tabular}{|c|c|c|}
\hline Sal & \%HR & Aw \\
\hline LiCl & 11.3 & 0.113 \\
\hline KF & 27.3 & 0.273 \\
\hline CoCl2 & 46.74 & 0.467 \\
\hline NaBr & 57.57 & 0.576 \\
\hline KI & 68 & 0.680 \\
\hline NaCl & 75.3 & 0.753 \\
\hline KCl & 84.43 & 0.843 \\
\hline
\end{tabular}

Se controló el peso de la muestra cada 5 días hasta obtener un peso constante (condición de equilibrio). Se consideró como condición de equilibrio cuando ya no hubiese variación dentro de los límites del equipo utilizado.

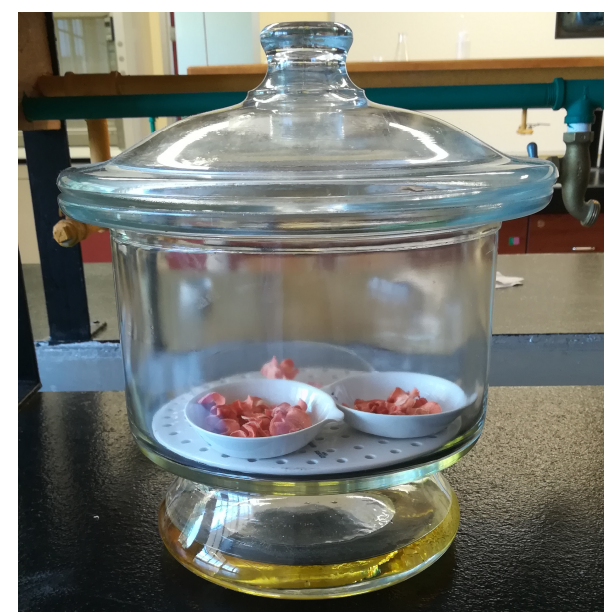

Figura 2. Desecador con las muestras y la solución de sal saturada.

Previo a los ensayos de humedad de equilibrio, se determinó por triplicado la humedad inicial de la zanahoria fresca y deshidratada utilizando una balanza de humedad marca Precisa modelo XM50. 


\subsection{Modelado de la isoterma de sorción}

Se graficó el contenido de humedad de equilibrio contra la actividad de agua para la obtención de las isotermas de sorción de la zanahoria. En la tabla 2 se presentan diferentes ecuaciones para modelar las isotermas de sorción, sin embargo, en esta investigación solo se trabajó con los modelos de BET, GAB y Henderson.

Los datos experimentales se trabajaron con estos modelos y se evaluó el mejor ajuste para producto elegido, mediante el coeficiente de correlación lineal $\left(\mathrm{R}^{2}\right)$ para determinar.

\section{Resultados y Discusión}

\subsection{Preparación de la muestra y deshidratado}

La humedad inicial de la zanahoria fresca fue de $0.8875 \mathrm{~g}$ de agua / $\mathrm{g}$ de producto. Luego del proceso de deshidratado se redujo a $0.1266 \mathrm{~g}$ agua / $\mathrm{g}$ de materia seca. En la figura 3 se muestra la pérdida de peso de la zanahoria durante la deshidratación en función del tiempo. Esta pérdida de peso es referente al agua que se elimina durante este tratamiento. Por lo tanto, se consigue una disminución del contenido de humedad, como se muestra en la figura 4 . Se puede observar que la mayor pérdida de agua ocurre durante las primeras 3.5 horas y se alcanza un peso constante a las siete horas utilizando una temperatura de $46^{\circ} \mathrm{C}$.

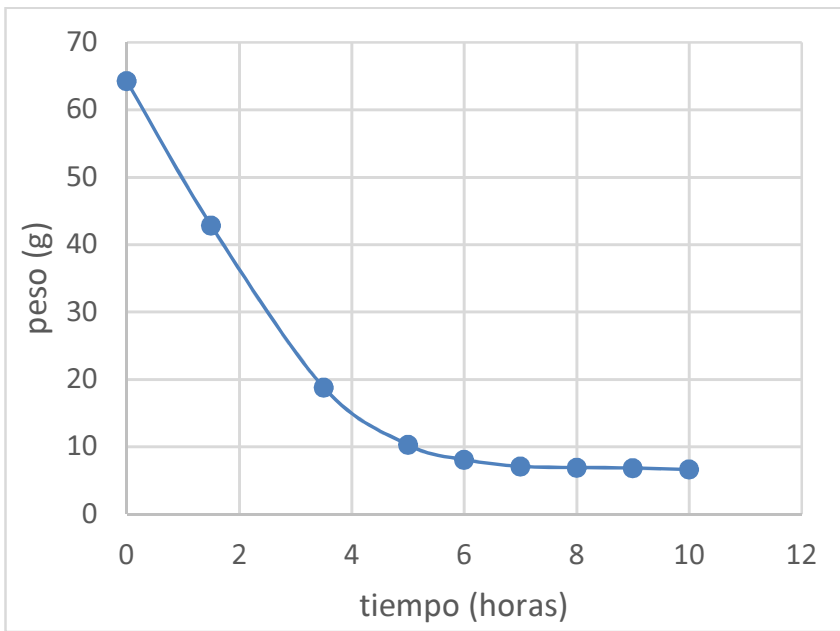

Figura 3. Pérdida de peso del producto fresco en función del tiempo durante el proceso de deshidratación.

Este comportamiento concuerda con los estudios realizados por Lee [13] y Sonmete, Menges, Ertekin y Özcan [14], quienes midieron las características de secado de rebanadas de zanahoria de diferentes espesores a temperaturas de $50^{\circ} \mathrm{C}$ y $55^{\circ} \mathrm{C}$, respectivamente. Las rebanadas de $3 \mathrm{~mm}$ de espesor alcanzaron la condición de equilibrio a las siete horas para la temperatura de $50^{\circ} \mathrm{C}$ y a las cuatro horas para la temperatura de $55^{\circ} \mathrm{C}$.

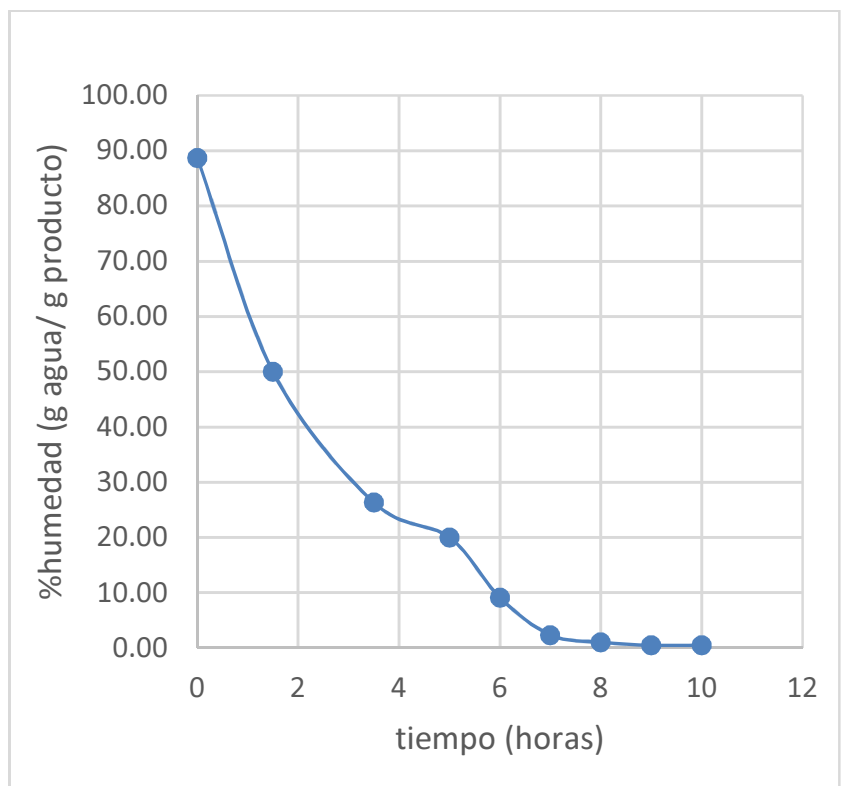

Figura 4. Disminución del contenido de humedad durante el proceso de deshidratado.

\subsection{Determinación de la isoterma}

En la figura 5 se muestran los datos de humedad de equilibrio en función de la actividad de agua a una temperatura de $22^{\circ} \mathrm{C} \pm 1{ }^{\circ} \mathrm{C}$. Se obtuvo una isoterma de tipo II, según la clasificación de Van der Waals, la cual es típica para alimentos como frutas y verduras [12].

Por otra parte, Eim, Rosselló, Femenia y Simal [15] obtuvieron las isotermas de zanahorias deshidratadas a cinco temperaturas diferentes y sus resultados arrojaron una isoterma tipo III, lo cual asociaron al alto contenido de azúcares y carbohidratos (cerca del $81 \%$ en materia seca) que se disolvieron y cambiaron la estructura cristalina del azúcar hacia un estado amorfo, aumentando así los sitios de adsorción.

\subsection{Modelado de la isoterma}

En la tabla 3 se presentan los resultados obtenidos para los modelos utilizados con sus respectivas constantes y valores de $\mathrm{R}^{2}$. De acuerdo con esto, los modelos que mejor se ajustaron al comportamiento de los datos experimentales fueron el modelo de BET y GAB, 
los cuales presentaron $\mathrm{R}^{2}$ de 0.975 y 0.994 , respectivamente.

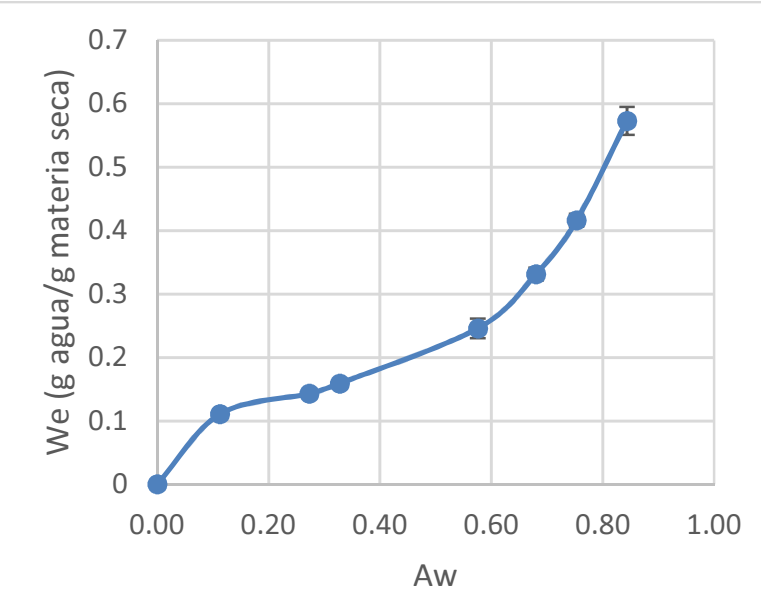

Figura 5. Contenido de humedad de equilibrio de las zanahorias deshidratadas a temperatura de $22{ }^{\circ} \mathrm{C}$.

Tabla 3. Valores de los parámetros de los modelos de sorción ajustados

\begin{tabular}{|c|c|c|}
\hline Modelo & Parámetro \\
\hline \multirow{4}{*}{ BET } & Wo & 0.102 \\
\cline { 2 - 3 } & $\mathrm{C}$ & 19.62 \\
\cline { 2 - 3 } & $\mathrm{R}^{2}$ & 0.975 \\
\hline \multirow{4}{*}{ GAB } & $\mathrm{k}$ & 0.944 \\
\cline { 2 - 3 } & $\mathrm{W}$ & 0.125 \\
\cline { 2 - 3 } & $\mathrm{C}$ & 90.50 \\
\cline { 2 - 3 } & $\mathrm{R}^{2}$ & 0.994 \\
\hline \multirow{3}{*}{ Henderson } & $\mathrm{F}$ & -2.909 \\
\cline { 2 - 3 } & $\mathrm{N}$ & 1.648 \\
\cline { 2 - 3 } & $\mathrm{R}^{2}$ & 0.920 \\
\hline
\end{tabular}

Estudios anteriores realizados sobre zanahorias bajo condiciones similares se presentan en la tabla 4 y son comparados con los datos obtenidos en esta investigación. Iglesias y Chirife estudiaron las propiedades de adsorción y desorción aplicando su ecuación para determinar la humedad de la monocapa obteniendo un $\mathrm{Wo}=0.052 \mathrm{~kg}$ de agua $/ \mathrm{kg}$ de materia seca para ambas isotermas. Por otro lado, Kiranoudis estudió solo el comportamiento de desorción aplicando el modelo de GAB, obteniendo un Wo de $0.21 \mathrm{~kg}$ de agua/kg de materia seca.
Los resultados obtenidos muestran los valores de humedad de la monocapa, Wo, encontrados utilizando los modelos de BET y GAB fueron de $0.102 \mathrm{~kg}$ de agua $/ \mathrm{kg}$ de m.s. y $0.125 \mathrm{~kg}$ de agua $/ \mathrm{kg}$ de m.s., respectivamente. Mientras que los valores de la constate de energía, C, fueron de 19.62 para BET y 90.50 para GAB. De acuerdo con la investigación de Timmermann, los valores de Wo obtenidos del modelo de BET siempre resultan menores que los obtenidos por el modelo de $\mathrm{GAB}$, contrariamente al parámetro $\mathrm{C}$, que se obtienen mayores valores con el modelo de BET que con el modelo de GAB [16].

Tabla 4. Valores de los parámetros de GAB obtenidos en estudios similares

\begin{tabular}{|c|c|c|c|c|}
\hline $\begin{array}{l}\text { Especificaciones } \\
\text { del producto }\end{array}$ & Wo & C & $\mathbf{k}$ & Fuente \\
\hline $\begin{array}{l}\text { Zanahorias } \\
\text { deshidratadas en } \\
\text { congelación a } \\
40^{\circ} \mathrm{C}\end{array}$ & 0.066 & 3.6 & 1.04 & $\begin{array}{l}\text { Lavelli et } \\
\text { al. [4] }\end{array}$ \\
\hline $\begin{array}{l}\text { Zanahorias } \\
\text { deshidratadas } \\
\text { con aire a } 37^{\circ} \mathrm{C}\end{array}$ & 0.052 & n.d. & n.d. & $\begin{array}{l}\text { Iglesias } \\
\text { \&Chirife } \\
{[17]}\end{array}$ \\
\hline $\begin{array}{l}\text { Zanahorias } \\
\text { frescas a } 37^{\circ} \mathrm{C}\end{array}$ & 0.052 & n.d. & n.d. & $\begin{array}{l}\text { Iglesias } \\
\text { \&Chirife } \\
{[17]}\end{array}$ \\
\hline $\begin{array}{l}\text { Zanahorias } \\
\text { frescas a } 45^{\circ} \mathrm{C}\end{array}$ & 0.21 & 3.9 & 0.66 & $\begin{array}{l}\text { Kiranoudis } \\
\text { et al. [18] }\end{array}$ \\
\hline $\begin{array}{l}\text { Zanahorias } \\
\text { deshidratadas } \\
\text { con aire a } 22^{\circ} \mathrm{C}\end{array}$ & 0.125 & 90.5 & 0.944 & $\begin{array}{l}\text { Este } \\
\text { trabajo }\end{array}$ \\
\hline
\end{tabular}

Wo es el contenido de humedad de la monocapa; C y k son parámetros de la ecuación de $\mathrm{GAB}$ relacionados con la temperatura; n.d.: no fue determinado.

En cuanto al valor de k, se obtuvo que fue de $0.944 \mathrm{y}$ se encuentra dentro del rango de los valores obtenidos en investigaciones anteriores. Igualmente, Timmermann establece que entre menor es el valor de $\mathrm{k}$, mayor son las diferencias entre los valores de Wo y $\mathrm{C}$ en ambos modelos, y que si $\mathrm{k}=1$, la ecuación del modelo de GAB se reduciría a la ecuación del modelo de BET [16].

En cuanto a los modelos de BET, GAB y Henderson aplicados, el que obtuvo el mayor valor de ajuste $\mathrm{R}^{2}$ fue el de GAB con 0.994. En la figura 6 se presentan las isotermas modeladas y la obtenida experimentalmente. 


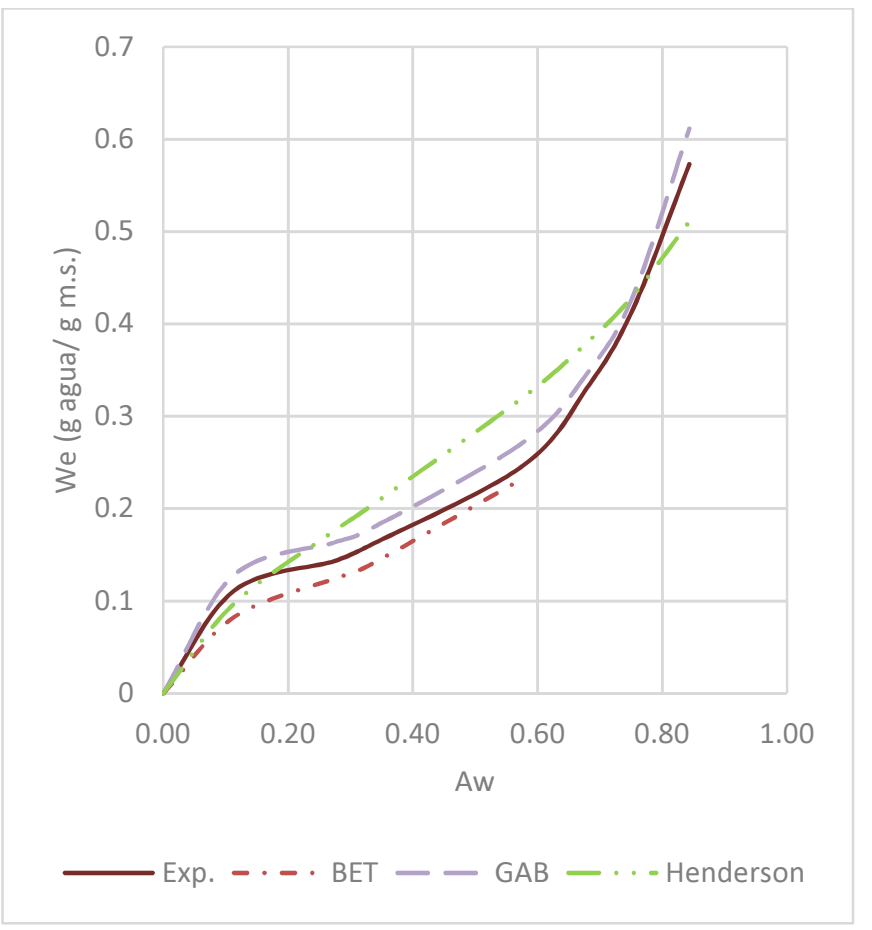

Figura 6. Isotermas modeladas y experimental.

\section{Conclusiones}

La isoterma de adsorción de humedad obtenida a $22^{\circ} \mathrm{C} \pm 1{ }^{\circ} \mathrm{C}$ para las zanahorias deshidratadas resultó ser sigmoidea de tipo II. De acuerdo con los resultados obtenidos en esta investigación, los valores de humedad de la monocapa, Wo, están en un intervalo de 0.102 a $0.125 \mathrm{~g}$ agua $/ \mathrm{g}$. m.s. Las tres ecuaciones utilizadas modelan satisfactoriamente los datos experimentales, sin embargo, los modelos de BET y GAB fueron los más adecuados por su alto grado de ajuste con $\mathrm{R}^{2}$ superiores a 0.97 , destacándose el modelo de GAB con 0.994 .

No se encontraron valores de estos parámetros de adsorción (Wo, C y k) a la temperatura de trabajo de esta investigación.

Según los resultados obtenidos, el $12.5 \%$ de humedad corresponde a la máxima humedad permitida para este producto a la temperatura estudiada. Donde las reacciones enzimática, no enzimática y oxidación son mínimas, permite mayor estabilidad del producto y una mayor vida de anaquel.

Es necesario realizar mayores estudios sobre este tema, ya que esta información es necesaria para predecir la correcta estabilidad durante el almacenado de los productos deshidratados a diferentes temperaturas.

\section{Referencias}

[1] MIDA, «Fichas Técnicas de Hortalizas Varias,» Panamá, 2015.

[2] FAO, «Productos frescos de verduras. Fichas Técnicas,» 2014.

[3] R. Monges y H. Campos, «Tabla de composición de Alimentos de Costa Rica,» INCIENSA, Costa Rica, 2013.

[4] Instituto de Nutrición de Centroamérica y Panamá, «Tabla de composición de Alimentos de Centroamérica,» 2012.

[5] S.-W. Lee, B.-K. Kim y J.-A. Han, «Physical and functional properties of carrots differently cooked within the same hardnessrange,» LWT - Food Science and Technology, vol. 93, pp. 346 $353,2018$.

[6] V. Lavelli, A. Zanibooni y B. Zanoni, «Rate of Carotenoid Degradation in Dehydrated Carrots,» 2006. [En línea]. Available: https://iufost.edpsciences.org/articles/iufost/pdf/2006/01/iufost06000 857.pdf. [Último acceso: 20 Enero 2019].

[7] S. B. Mariem y S. B. Mabrouk, «Moisture sorption isotherms and isoteric heat of sorption of Tomato Slices,» American Journal of Renewable and Susteinable Energy, pp. 140-155, 2015.

[8] R. Andrade, R. Lemus y C. Pérez, «Models of Sorption Isotherms for Food: Uses and Limitations,» Revista de la Facultad de Química Farmacéutica, vol. 18, nº 3, pp. 325 - 334, 2011.

[9] E. Soteras, J. Gil, P. Yacanto, S. Muratona, C. Abaca y M. Sustersic, «Isotermas de adsorción y desorción de agua en Leche en Polvo II. Leche entera,» Avances en Ciencia e Ingeniería, pp. 57 66, 2014.

[10] J. V. García Pérez, Contribución al Estudio de la Aplicación de Ultrasonidos de potencia en el Secado Convectivo de Alimentos, Universidad Politécnica de Valencia, 2007.

[11] N. Martínez Navarrete, Termodinámica y cinética de sistemas: alimento - entorno, España: Editorial de la Universitat Politécnica de Valencia, 1998

[12] J. E. Zapata, Ó. A. Quintero y L. D. Porras, «Isotermas de Sorción para Avena (Avena sativa) en grano,» Revista Agron, vol. 23, pp. 82 $-92,2015$.

[13] G. Lee, «Drying Characteristics of Carrot and Green Pumpkin Slices in Waste Heat Dryer,» Journal of Biosystems Engineering, vol. 37, pp. $36-43,2012$.

[14] M. Sonmete, H. Menges, C. Ertekin y M. Özcan, «Mathematical modeling of thin layer drying of carrot slices by forced convection,» Food Measure, vol. 11, pp. 629 - 638, 2017.

[15] V. Eim, C. Rosselló, A. Femenia y S. Simal, «Moisture Sorption Isotherms and Thermodynamic Properties of Carrot,» International Journal of Food Engineering, vol. 7, n 3, 2011.

[16] E. Timmermann, «Multilayer sorption parameters: BET or GAB values?,» Colloids and Surfaces A: Physicochemical Engineering Aspects, vol. 220, pp. 235 - 260, 2003. 
[17] H. A. Iglesias y J. Chirife, Handbook of food isotherms: Water sorption parameters for food and food components, New York: Acdemic Press, 1982.

[18] C. T. Kiranoudis, Z. B. Maroulis, E. Tsami y D. Marinos-Kouris, «Equilibrium moisture content and heat of desorption of some vegetables,» Journal of Food Engineering, vol. 20, pp. 55 - 74, 1993. 